\title{
Potential application of cold atmospheric plasma jet in the treatment of onychomycosis
}

\section{AC Borges, TMC Nishime, GMG Lima, KG Kostov, CY Koga-Ito*}

São José dos Campos Institute of Science and Technology and Guaratinguetá Faculty of Engineering; São

Paulo State University (UNESP), Brazil.

\section{OBJECTIVE}

This study aimed to evaluate the effects of cold atmospheric plasma (CAP) on Trichophyton rubrum growth, germination, adherence and infection capacity.

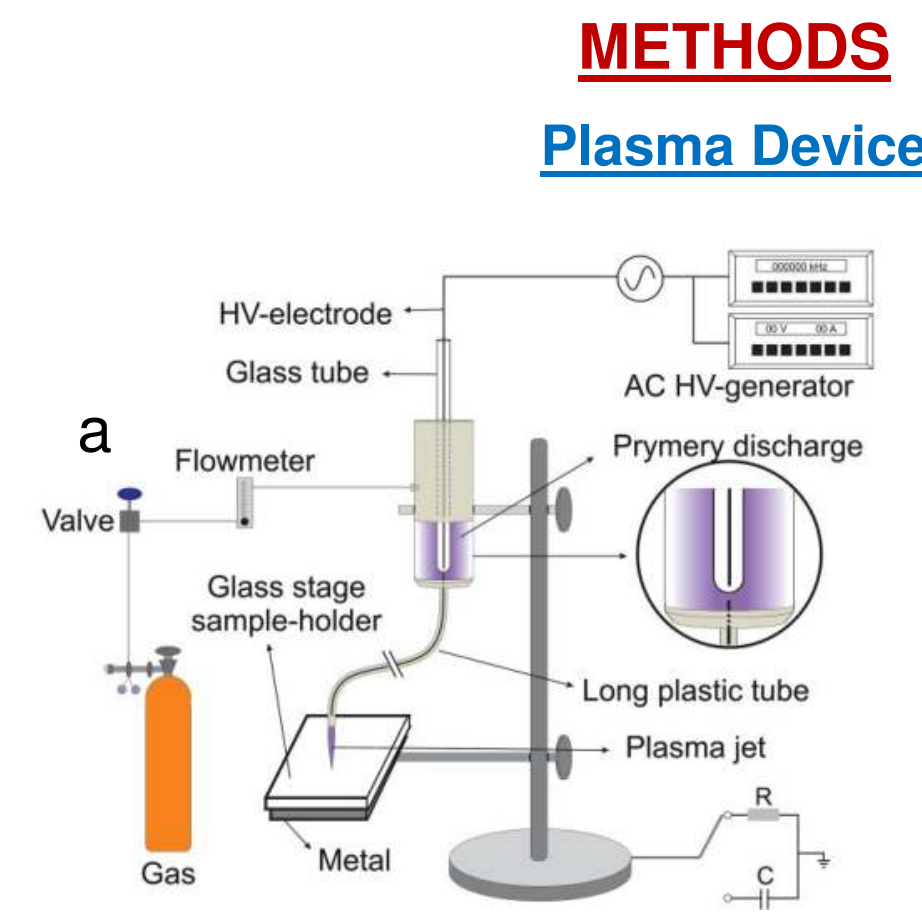

Operating gas:

Helium (99.5\% purity)

Flow rate: 2.0 SLM

Signal frequency:

$32.0 \mathrm{kHz}$

Signal period: $1.5 \mathrm{~ms}$

Power: $1.0 \mathrm{~W}$

\section{Effect of CAP on conidia suspension}

Trichophyton rubrum (ATCC 28189)/1.0 x 106 conidia $\mathrm{ml}^{-1}$

Exposition to $\operatorname{CAP}(5,10$ and $15 \mathrm{~min}$ ) - Growth observation in RPMI broth $/ 28^{\circ} \mathrm{C}$ (turbidity) from 24 to $144 \mathrm{~h}$

\section{Effect of CAP on mycelial growth}

T. rubrum /Potato dextrose agar (PDA) for 7 days $\left(28^{\circ} \mathrm{C}\right)$

$1.0 \times 10^{6}$ conidia $\mathrm{ml}^{-1} \Longrightarrow$ PDA $\Longrightarrow 48 \mathrm{~h}\left(28^{\circ} \mathrm{C}\right)$

Exposition to $\operatorname{CAP}(5,10$ and $15 \mathrm{~min})$

Mycelial growth measurements (2, 5 7, 12 and 15 days)

Effect of CAP on conidia adherence and infection

Ex vivo nail infection model (Ethics Committee CONEP 31787814.1.0000.0077)

T. rubrum $\left(1.0 \times 10^{6}\right.$ conidia $\left.\mathrm{ml}^{-1}\right) \quad$ Human nail specimens

Treated with CAP

$5,10,15 \min$

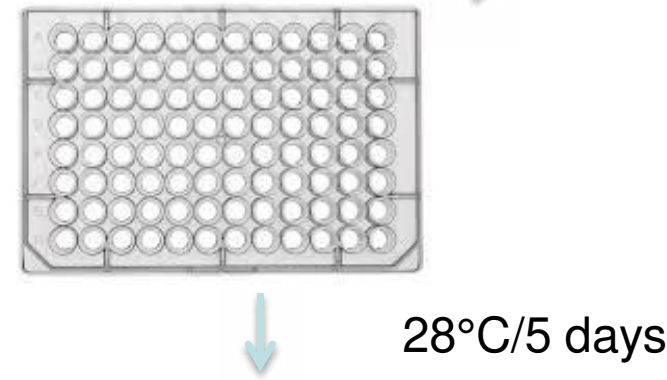

inverted optical microscope/SEM

\section{RESULTS}

Effect of CAP on conidia suspension

\begin{tabular}{|c|c|c|c|c|}
\hline \multicolumn{5}{|c|}{ Growth of $T$. rubrum in RPMI medium } \\
\hline & CONTROL & $5 \mathrm{~min} / \mathrm{CAP}$ & $10 \mathrm{~min} / \mathrm{CAP}$ & $15 \mathrm{~min} / \mathrm{CAP}$ \\
\hline $24 \mathrm{~h}$ & + & - & - & - \\
\hline $48 \mathrm{~h}$ & ++ & + & - & - \\
\hline $72 \mathrm{~h}$ & +++ & ++ & + & - \\
\hline $96 \mathrm{~h}$ & +++ & +++ & + & - \\
\hline $144 \mathrm{~h}$ & +++ & +++ & ++ & + \\
\hline
\end{tabular}

Effect of CAP on mycelial growth
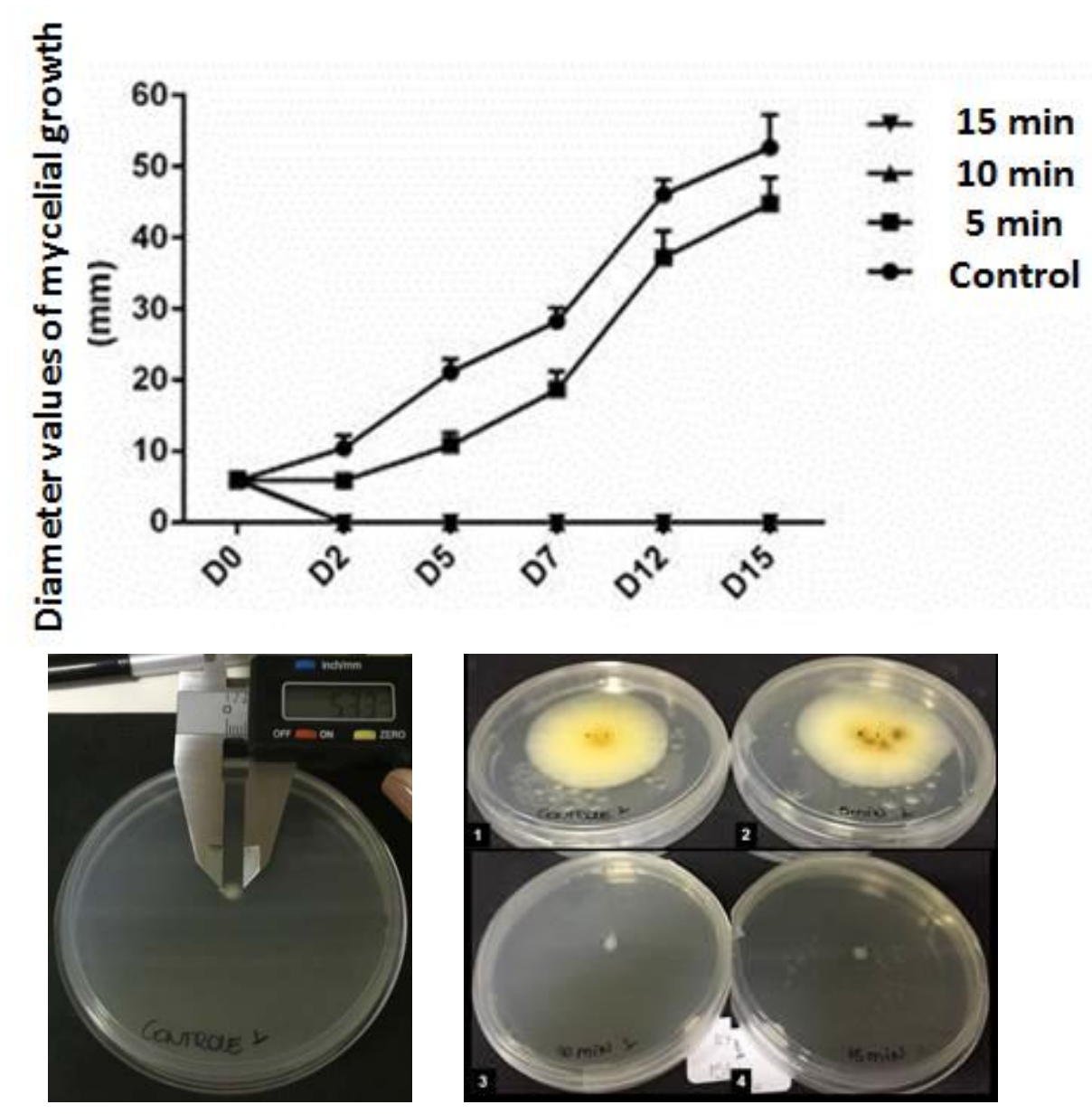

Mean diameter values of mycelial growth control: $52.69 \mathrm{~mm}$; $5 \mathrm{~min}: 44.48 \mathrm{~mm}$; $10 \mathrm{~min}: 0 \mathrm{~mm} ; 15 \mathrm{~min}: 0 \mathrm{~mm}$

Effect of CAP on conidia adherence and infection CAP for 10 and 15 minutes $\longrightarrow$ no infection

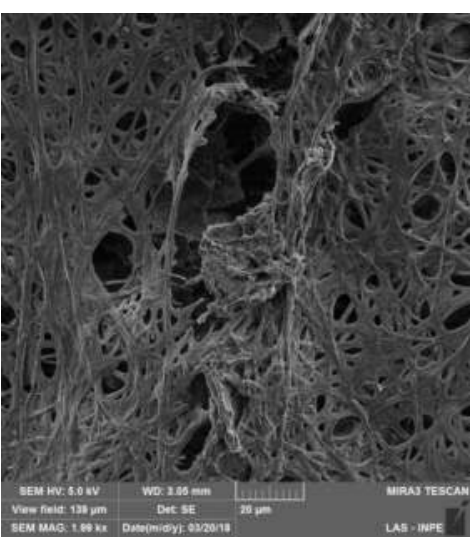

Control

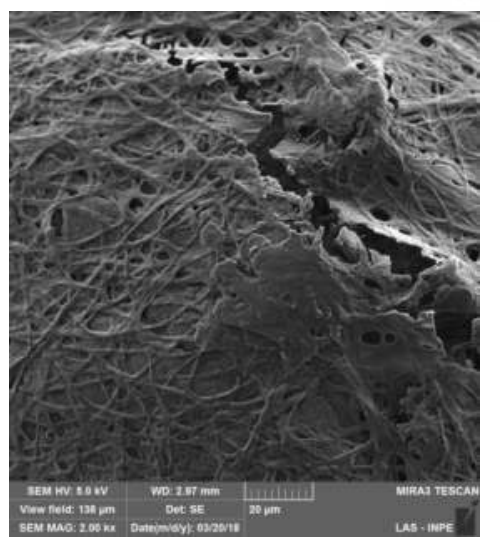

$5 \mathrm{~min}$

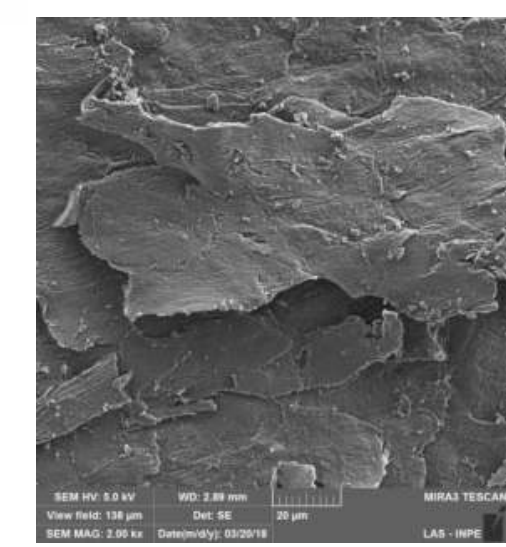

$15 \min$

\section{CONCLUSION}

A single exposure to CAP for 15 minutes was able to inhibit fungal growth, adherence and infection capacity. CAP can be a promising therapeutic alternative to the treatment of onychomycosis. 